\title{
Do we sing from the same hymn sheet? Peri-operative management of hip fractures - a survey.
}

\section{R Sinha ${ }^{1}$, T Fletcher ${ }^{1}$, N Bedforth ${ }^{2}$}

1. Senior Fellow - Regional Anaesthesia. 2. Honorary Associate Professor and Consultant Anaesthetist - Nottingham University Hospitals NHS Trust.

The peri-operative management of patients presenting with hip fractures has been the focus of much attention with several national and international guidelines published in the previous years. 1,2,3 These patients can provide a challenge to the surgical and anaesthetic teams as they are often elderly, with multiple comorbidities as well as ongoing issues relating to the events around the occurrence of the injury such as falls etc., which leaves them at high risk for peri-operative morbidity and mortality. We aimed to survey trauma anaesthetists at a busy major trauma centre with a view to developing uniformity of practice.

\section{Methods:}

We developed a survey outlining the three most common type of patients with a hip fracture seen in our trauma centre i.e., the middle-aged patient with an unexpected significant injury; the slightly older patient with co-morbidities; and the elderly, frail patient with several serious medical co-morbidities. The survey included questions about preferred anaesthetic technique, method of intra-operative analgesia, use of sedation and postoperative analgesic techniques.

The survey was administered to all anaesthetists with a regular trauma list over a two week period.

\section{Discussion:}

Following on from the development of several guidelines previously alluded to, hip fracture management has been standardised to a large extent with significant input from the multi-disciplinary team leading to improving outcomes.

Although we do not have a departmental guideline, our survey demonstrates that for the large part, we have relatively uniform practice in our unit. This is likely to be due in part to the trickle down effect of national guidelines and to the work done by our academic department.

We did demonstrate some differences between anaesthetists in the management of specific patients; this is likely to be due to the interpretation of information provided and is representative of the viewpoint that there is often more than one 'correct way' of doing things.

\section{Conclusion:}

Overall we demonstrated relatively uniform practice as a unit and writing a consensus guideline based on these viewpoints is feasible and would be helpful to new starters in our department.

\begin{tabular}{|c|c|c|c|}
\hline & Case I & Case 2 & Case 3 \\
\hline Preferred anaesthetic technique & GA (13\%), Spinal (73\%), either (14\%) & GA (6\%), Spinal (73\%), Either (20\%) & GA (33\%), Spinal (53\%), Either (14\%) \\
\hline Anaesthetic agent in spinal & Heavy Bupivacaine (92\%), Isobaric (8\%) & Heavy Bupivacaine (85\%), Isobaric (15\%) & Heavy Bupivacaine (80\%), Isobaric (20\%) \\
\hline Spinal opiates & $60 \%$ & $46 \%$ & $14 \%$ \\
\hline Intra-operative sedation & $61 \%$ & $35 \%$ & $60 \%$ \\
\hline NSAIDs & $100 \%$ & $100 \%$ & $100 \%$ \\
\hline Long acting opiates & $40 \%$ & $33 \%$ & $13 \%$ \\
\hline Short acting opiates & $100 \%$ & $100 \%$ & $100 \%$ \\
\hline
\end{tabular}

Table 1: Summary of survey

Results:

References:

15 anaesthetists were surveyed in total of which 11 were consultants, two staff grades and two senior anaesthetic registrars. 13 used femoral nerve blocks and two used fascia iliaca blocks to provide intra-operative and post-operative analgesia. All of these were delivered using ultrasound guidance.

The salient features of the survey are summarised in Table 1. Of particular interest are the high rate of concordance with respect to anaesthetic technique of choice for the first two patient types.

1. Hip fracture in adults. National Institute of Clinical Excellence. 2011.

2. Management of proximal femoral fractures. AAGBI. 2012.

3. Australia and New Zealand guideline for hip fracture care. Australia and New Zealand hip fracture registry steering group. 2014. 